\title{
Occupational Exposure to Solar Radiation in Australia: Who is Exposed and What Protection Do They Use?
}

\begin{abstract}
Objective: Solar ultraviolet radiation (UVR) exposure is widely recognised as a leading cause of skin cancer, with outdoor workers being particularly at risk. However, very little is known on a national level about how many workers are actually exposed to solar radiation, the circumstances in which they are exposed, or their use of protective measures.
\end{abstract}

Methods: The Australian Work Exposures Study (AWES) was a cross-sectional telephone survey of 5,023 Australian workers aged 18 to 65 . A subset of 1,113 respondents who indicated that they worked outdoors was asked about their exposure to solar radiation in terms of the amount of time they spent working outdoors, their working location, and their use of sun protective measures.

Results: A total of 1,100 respondents (22\% overall) were assessed as being exposed to solar radiation at work. Exposure was more likely among males and those residing in lower socioeconomic and regional areas. Sun protection was used by $95 \%$ of the respondents, although the level of protection varied among workers, with only $8.7 \%$ classified as fully protected.

Conclusions: This study provides valuable information regarding solar exposure which has not previously been available.

Implications: The results of this study will inform strategies for risk reduction. 


\section{Introduction}

The International Agency for Research on Cancer has classified solar ultraviolet radiation (UVR) as a human carcinogen, noting that there is sufficient evidence for its causal involvement in melanoma and non-melanoma skin cancers, including squamous cell carcinoma and basal cell carcinoma. ${ }^{1}$ Indeed, solar UVR exposure is widely recognised as a leading cause of skin cancer, with a World Health Organisation report suggesting that around $50-90 \%$ of melanomas and basal cell carcinomas are attributable to sun exposure, along with $50-70 \%$ of squamous cell carcinomas in light-skinned populations. ${ }^{2}$ The proportion of skin cancers attributable to solar UVR is even higher in Australia, because of its equatorial proximity, clear atmospheric conditions, and loss of ozone in spring and early summer. ${ }^{3-4}$ These factors, combined with the largely light-skinned population, contribute to Australia having the highest incidence of skin cancer in the world. ${ }^{4-5}$

The workplace is a significant setting in which UVR exposure occurs for a large number of Australians, with those particularly at risk including building and construction workers, farming and horticultural workers, landscape and gardening workers, physical education teachers and outdoor sports coaches, and mining and resource workers. ${ }^{6}$ It has been estimated that outdoor workers in Queensland receive on average about twice as much UVR over a two-day period as schoolchildren or home workers, ${ }^{7}$ while outdoor workers in Perth were found to be exposed to six to eight times as much UVR as classroom teachers. ${ }^{8}$ Significant exposures may also be experienced by workers who spend less extended periods of time outdoors, ${ }^{9}$ with a Queensland study finding that $77 \%$ of building inspectors and $40 \%$ of painters were exposed to UVR exceeding the occupational exposure limits of $30 \mathrm{~J} / \mathrm{m}^{2}$ per eight-hour working day. ${ }^{3}$

Despite the significant contribution of outdoor work to the total amount of solar UVR exposure, and the particular relevance of this to Australian conditions, very little 
comprehensive information is available concerning the actual extent of exposure among the working population. Most studies have concentrated on one industry or job title. One Australian study, the National Hazard Exposure Worker Surveillance (NHEWS), found that $34 \%$ of respondents worked in direct sunlight for at least part of their working day. ${ }^{10}$ This survey was however limited to workers from predetermined priority industries, meaning that it was not representative of the general Australian working population. Additionally, NHEWS relied on workers' self-reported perception of their exposure to sunlight, which may not necessarily reflect the actual level of solar UVR exposure experienced by workers. Further, exposure to solar UVR depends on many factors other than simply direct sunlight, including the geographic location, presence of reflective surfaces, and use of protective measures. ${ }^{6}$ However, very little information is as yet available concerning the circumstances in which outdoor workers are exposed as well as their use of protective measures.

We aimed to gain a nationally representative view of the prevalence of exposure to solar UVR among Australian workers, taking into account the protective measures used and the circumstances of exposure.

\section{Methods}

\section{Study Population}

This study was undertaken as part of a larger nationwide survey, the Australian Work Exposures Study (AWES), which investigated the current prevalence of occupational exposure to 38 carcinogens, including solar UVR, among Australian workers. ${ }^{11}$ Ethics approval for this study was obtained from the University of Western Australia Human Research Ethics Committee.

The sample for AWES was randomly drawn from a list of approximately 6 million Australian households and obtained from a commercial survey sampling firm. This list consisted of 
household contact details compiled from various public domain data sources such as telephone directories. Individual matching to the electronic White Pages revealed that approximately $18 \%$ of these households were not listed in the traditional telephone directory. Within households, currently employed residents aged between 18 and 65 were eligible to participate. The eligible person within each household who had the next birthday was selected for the interview. Up to ten attempts were made to contact each household before the designating it as unreachable. Calls were generally made outside of traditional working hours and across all days of the week in order to maximise the number of potentially eligible people contacted, and appointments were made for call-backs if the selected eligible person within the household was not available at the time of initial contact.

Of the 19,896 households telephoned during the course of AWES, 2,452 did not respond, 1,936 refused to participate, and 10,485 were ineligible. Reasons for ineligibility included not fitting study criteria (i.e. not aged between 18 and 65 years and/or not in current employment; $n=9,068)$, State quota being full $(n=906)$, poor English language ability $(n=388)$, and being too ill or deaf to participate $(n=123)$. Five thousand and twenty three interviews were completed, resulting in a response fraction (completed interviews $\div$ eligible and unknown households) of $53 \%$ and a cooperation fraction (completed interviews $\div$ eligible households) or $72 \%$. Thirty respondents provided insufficient data and were thus excluded from analysis, resulting in a final sample of 4,993 respondents.

\section{Data Collection}

All interviews were conducted by trained interviewers using computer-assisted telephone interviews (CATI). Respondents provided oral informed consent prior to any data being collected. Demographic information collected included age, gender, postcode of residence, country of birth, language spoken at home, and highest level of education. In addition, socioeconomic status (SES) and remoteness were determined by applying the Australian 
Bureau of Statistics Socio-Economic Indexes for Areas Index of Relative Socio-economic Disadvantage (ABS SEIFA IRSD) ${ }^{12}$ and Australian Standard Geographical Classification Accessibility/Remoteness Index of Australia (ASGC ARIA+) ${ }^{13}$ to the respondent's postcode.

The respondent's main job was then categorised as either potentially exposed or unexposed to any of the 38 carcinogens ${ }^{14}$ by the use of a simple screening tool. Respondents whose job fitted into one of 13 predetermined categories of unexposed jobs (e.g. white-collar professional, customer service) were classified as unexposed and their interview was ended. A total of 2,532 respondents were categorised as unexposed at this point. Basic job information (job title, main tasks at work, industry, frequency of work in terms of hours per week and weeks per year) was then collected from the remaining 2,491 respondents with the aim of using this information to assign them into one of 58 job-specific modules (JSMs). These modules included questions about the completion of tasks likely to involve exposure to carcinogens, and were developed by a team of occupational hygienists and epidemiologists. Twenty seven of these modules included questions related to solar UVR exposure. A set of standard questions was developed based on Safe Work Australia's NHEWS survey ${ }^{10}$ and the Australian Radiation Protection and Nuclear Safety Agency's (ARPANSA) UV guidance note. ${ }^{15}$ These questions collected information regarding the amount of time spent working outdoors (defined as less than one hour, between one and four hours, or more than four hours per day), the location in which work was conducted, the presence of reflective surfaces, and the use of protective measures including hats, sunscreen, protective clothing, and shade (defined as less than or more than half of the outdoor working time). A total of 1,113 respondents were assigned one of these 27 modules and thus completed these questions. All modules were completed using OccIDEAS, an online tool to manage interviews and exposure assessments. ${ }^{16}$ Each full interview took approximately 15 minutes. Following the 
interviews, each job was coded according to the Australian and New Zealand Standard Classification of Occupations (ANZSCO) ${ }^{17}$ and then categorised into one of 30 occupational groups, with each group containing occupations which were judged to be relatively homogenous in terms of exposure to the carcinogens of interest to this study. ${ }^{11}$

\section{Exposure Assessment}

Automatic assessments of the probability ('none' or 'probable') and level ('low', 'medium' or 'high') of exposure to solar UVR were derived within OccIDEAS using predetermined rules that had been developed on the basis of expert opinion and the ARPANSA UV guidance note. ${ }^{15}$ These rules took into account the amount of time spent working outdoors and the level of protection, with full protection defined as using all four protective methods (hat, sunscreen, clothing, shade) for more than half of the outdoor working time. Level of exposure was only downgraded if full protection was used. For example, someone working outdoors for more than four hours per day without adequate protection was assigned a high level of exposure, whereas someone working outdoors for the same amount of time but using all four protective methods for most of their working time was assigned a medium level. Accordingly, a low level of exposure was assigned both where a worker spent less than an hour working outdoors with inadequate protection, and where workers spent between one and four hours outdoors with full protection. All automatic assessments were reviewed by project staff for consistency.

\section{Statistical Analysis}

All statistical analyses were conducted using Stata version $12 .{ }^{18}$ A dichotomous measure of exposed or not exposed was used for the main analysis. Assessments were extrapolated with reference to the 2011 Census ${ }^{19}$ to calculate an estimate of the number of Australian workers currently exposed to UVR at work. These extrapolations were stratified by gender and 
conducted separately by occupational group in order to account for potential differences in exposure.

Logistic regression analyses were used to estimate odds ratios (ORs) and 95\% confidence intervals (CIs) exploring which demographic variables were associated with exposure, as well as to investigate the associations between the use of various sun protection methods and the circumstances of exposure. In addition, multinomial logistic regression was used to estimate relative risk ratios (RRs) and 95\% CIs to explore the variables associated with the use of sun protection. For the purposes of this analysis, inadequate sun protection was divided into none (using no form of sun protection for more than half of the outdoor working time) and some (using one to three forms of sun protection more than half of the outdoor working time), while full sun protection was defined as in the exposure assessment. All regression analyses were mutually adjusted.

For the purposes of analysis, outdoor workers were assigned into one of three groups based on the latitude of their working location and the annual average UV index of that location ${ }^{20}$ (see Figure 1): those working in areas with an average UV index greater than 11 (latitude above $18^{\circ} \mathrm{S}$ ); those in areas with an average UV index between 8 and 11 (latitude between $18^{\circ} \mathrm{S}$ and $30^{\circ} \mathrm{S}$ ); and those in areas with an average UV index less than 8 (latitude below $\left.30^{\circ} \mathrm{S}\right)$. A total of 88 respondents provided insufficient information on their working location and were therefore unable to be assigned into one of these groups.

Figure 1 


\section{Results}

\section{Exposure to Solar UVR}

Overall 1,100 respondents (22.0\%) were assessed as being exposed to solar UVR at work. The majority of these workers $(n=629,57.2 \%)$ were exposed at a high level, with 349 $(31.7 \%)$ exposed at a medium level and 122 workers (11.1\%) at a low level.

After adjusting for occupational group, exposure to solar UVR was found to be more likely among male compared with female workers, as well as among those residing in lower as opposed to higher SES areas and those in regional or remote areas rather than major cities (Table 1). There was also a significant trend for remoteness, whereby likelihood of exposure decreased with remoteness. The trend for SES was not significant. Those with a bachelor degree or higher were less likely than those who had only completed high school to be assessed as being exposed to solar UVR in the workplace.

\section{Table 1}

Exposure to solar UVR varied by occupational group and was highest in farmers, painters, plumbers, heavy vehicle drivers, animal and horticultural workers, and handypersons (Table 2). Within the same broad occupational groups, women tended to have lower prevalence of exposure than men.

\section{Table 2}

Upon extrapolation of these figures to the Australian working population based on occupational group prevalence, approximately 1,737,000 males (37.0\%, 95\% CI 35.7-38.3) and 335,000 females $(7.9 \%, 95 \%$ CI $7.2-8.7)$, or $2,072,000$ workers overall $(23.1 \%, 95 \%$ CI 21.9-24.2) were estimated to be exposed to solar UVR at work. 


\section{Circumstances of Exposure}

The majority of solar UVR exposed workers $(n=624,56.9 \%)$ spent more than four hours working outside, while 154 (14.0\%) worked outside for less than one hour. The amount of time spent working outdoors varied significantly by occupational group $(p<0.001)$, with farmers, plumbers, animal and horticultural workers, heavy vehicle drivers, and miners being more likely to work outside for longer periods of time.

In terms of latitude, the majority of exposed respondents $(n=746,73.9 \%)$ reported working in areas with an annual average UV index of less than 8 (latitude more than $30^{\circ} \mathrm{S}$ ). Only 27 workers $(2.7 \%)$ worked in areas with an annual UV index of greater than 11 (latitutde less than $18^{\circ} \mathrm{S}$ ). Latitude of working location varied significantly by occupational group ( $p=$ 0.048), with engineers, carpenters, animal and horticultural workers, and outdoor workers not elsewhere classified (NEC; e.g. sports instructors, deck hands) being more likely to work in areas with a latitude less than $18^{\circ} \mathrm{S}$. No differences were observed in terms of time spent working outdoors $(p=0.089)$.

The reflective surfaces that exposed workers reported working near included water $(n=227$, $20.6 \%)$, roofing iron $(n=207,18.8 \%)$, glass $(n=174,15.8 \%)$, and sand $(n=155,14.1 \%)$, with a total of 510 workers (46.5\%) reporting working near reflective surfaces. The presence of reflective surfaces differed significantly by time spent working outdoors $(p=0.006)$, whereby those working outside for greater than four hours were more likely to work near reflective surfaces, as well as by occupational group $(p<0.001)$, with tradespeople (including painters, plumbers, carpenters, construction workers, and electrical workers), miners, machine operators, and outdoor workers NEC more likely to work near reflective surfaces. 


\section{Use of Sun Protection}

A total of 1,056 respondents (94.9\%) used at least one form of sun protection for more than half of their outdoor working time, with protective clothing (used by $80.4 \%$ ) and hats (72.2\%) being used most frequently. Ninety-seven respondents $(8.7 \%)$ were classified as being fully protected (i.e. used all four methods for more than half of the time they spent working outdoors).

Age, gender, occupational group, hours spent working outdoors, latitude of working location, and presence of reflective surfaces were all entered as potential predictors of the use of sun protection. After mutual adjustment, the only significant predictors to emerge were age and occupational group. Specifically, those aged between 35 and 54 ( $R R=2.4,95 \%$ CI 1.2-4.9) were more likely than those aged under 35 to use full as opposed to some sun protection. In addition, farmers $(\mathrm{RR}=18.1,95 \% \mathrm{CI} 1.6-207.3)$ were significantly more likely than office workers to use full as opposed to no sun protection, while scientists $(\mathrm{RR}=5.9,95 \%$ CI 1.3 26.8), miners ( $\mathrm{RR}=7.2,95 \% \mathrm{CI} 1.1-48.2)$ and handypersons $(\mathrm{RR}=4.9,95 \% \mathrm{CI} 1.2-19.0)$ were significantly more likely than office workers to use full as opposed to some sun protection.

After adjusting for age, gender, and occupational group, the use of a hat was more likely among those who worked outdoors for more than four hours per day compared with those who worked outdoors for fewer hours, as well as among those who worked in the midlatitudes (compared with those who worked either above $30^{\circ} \mathrm{S}$ or below $18^{\circ} \mathrm{S}$ ) (Supplementary Table 1). Conversely, working in the shade was less likely among those who worked outdoors for more than four hours than those who worked outdoors for between one and four hours. 


\section{Discussion}

We investigated the current prevalence of occupational exposure to solar UVR and the use of protective measures among Australian workers. Overall, 22\% of respondents were assessed as being exposed to solar UVR in the workplace, with exposure being more likely among males and those residing in lower SES and regional areas after controlling for occupational group. When these figures were extrapolated to the Australian working population, approximately $23.1 \%$ of all workers were estimated to be exposed to solar UVR at work. Not surprisingly, this is higher than estimates obtained in Canada $(8.8 \%)^{21}$ and the United Kingdom (13.7\%), ${ }^{22}$, although it is somewhat lower than the estimate of $34 \%$ obtained in a survey conducted by Safe Work Australia in 2008 (NHEWS). ${ }^{10}$ This disparity is probably due to the different sampling frames and exposure assessment methodologies used. Our study included a cross-section of workers across all industries, while NHEWS targeted specific priority industries. In addition, whereas we used task-based questions to determine exposure, taking into account the use of protection and the amount of time respondents spent working outdoors, NHEWS asked workers expressly about their exposure to direct sunlight at work. This self-reported perception may not necessarily reflect the actual exposure to solar UVR experienced by workers, particularly as this is known to vary depending on other factors such as the use of protective measures, and thus it may be argued that the method used in our study provides a more accurate estimate of the prevalence of solar UVR exposure.

This study also investigated the circumstances of workers' exposure to solar UVR in terms of the presence of reflective surfaces and average UV index of the working location. Approximately $46 \%$ of respondents reported working near reflective surfaces including water, roofing iron, and glass. Exposure to these surfaces is likely to contribute significantly to the amount of UVR these workers receive. Different surfaces reflect varying amounts of solar UVR. ${ }^{23}$ For example, galvanised roofing surfaces and sand have been shown to reflect 
up to $30 \%$ of the available UVR, ${ }^{24-25}$ while asphalt reflects approximately $10 \%$ and water $9 \% .{ }^{23}$ Therefore workers may be exposed to solar UVR both from direct sunlight and through reflection from surfaces in their working environment. ${ }^{15}$ Those who spend a considerable portion of their day inside a vehicle can also receive substantial amounts of UVR, due to both reflection and refraction through glass. ${ }^{5}$

The geographical location, or more specifically the latitude of the location where work takes place, has also been shown to be an important factor influencing exposure to solar UVR. ${ }^{6}$ The amount of available ambient UVR varies inversely with latitude, ${ }^{4,20}$ such that areas in the lower latitudes (closer to the equator) have higher levels of ambient UVR than areas in the higher latitudes, and therefore more potential for exposure which exceeds recommended limits. ${ }^{15}$ Only a minority of respondents in the current study $(2.7 \%)$ reported working in areas with an extreme average UV index (above 11), although this differed by occupational group with engineers, carpenters, animal and horticultural workers, and outdoor workers NEC being more likely to work in these areas. These workers may therefore be at greater need for adequate sun protection, ${ }^{15}$ although we did not find these groups to use sun protection to a greater degree than other occupational groups.

Various sun protection methods are available, including sunscreen, protective clothing, hats, and sunglasses. ${ }^{26-27}$ Sun protection was used by the large majority of respondents in the current study, with the most frequently used method being protective clothing and hats, consistent with previous research. ${ }^{3,28}$ For example, in a Canadian study, 58\% of workers reported wearing a hat at work, while $60 \%$ wore sun protective clothing. ${ }^{28}$ Among Queensland construction workers, 39\% of workers were observed to wear an appropriate head covering and $62 \%$ to wear adequately protective clothing, while $30 \%$ used sunscreen and 50\% wore sunglasses. ${ }^{3}$ The use of higher level controls, such as working in the shade and avoiding sunlight during times of high UV irradiance, has been found to be less common, as 
these techniques may be incompatible with work requirements. ${ }^{15}$ Compounding the issue is the fact that no single protective measure provides complete protection against UVR exposure, with the exception of complete avoidance of sunlight, and thus the combination of various measures is necessary. ${ }^{29}$ However, only a minority of workers in the current study (8.7\%) were classified as being fully protected, defined as using protective clothing, a hat, sunscreen, and working in the shade for more than half of the time spent working outdoors. This is similar to findings among Queensland construction workers, where $10 \%$ of workers were found to be appropriately protected, defined in that study as wearing a hat, collared shirt, and sunscreen. ${ }^{3}$

There are limitations to the approach used in this study. For example, these findings may be biased due to the potential non-representativeness of the original sample; for example, by not including those too poor or itinerant to have a telephone. This is a limitation of using commercial samples, as the original sampling frame is somewhat unknown. There is also a potential bias resulting from the comparatively high number of non-responders, refusals, and poor English speakers, although the impact of this bias is not known as no additional information is available regarding these non-participants. Further, information regarding the time of day when workers were exposed was not collected, and was therefore not taken into account in the exposure assessments. However, respondents were asked to provide information about the number of hours they spent working outdoors during the day, and this information was used in the exposure assessments. While levels of solar UVR are known to be at their highest during the middle parts of the day (10.00am to $2.00 \mathrm{pm})$, significant exposures are still possible outside of these times, and sun protection is recommended to be used whenever the UV index is three or above, meaning that, particularly in the summer months, protection may be required across the majority of the day in Australia. ${ }^{29}$ Therefore it 
is likely that hours of outdoor work, as used in these assessments, may be a sufficient measure in lieu of time of day information.

This study provides new insights regarding occupational exposure to solar UVR and the use of protective measures among workers. The findings of this study are likely to have important implications for workplace policy and practice. Our results show that, although solar UVR exposure is a well-recognised and established carcinogen, a significant proportion of Australian workers are still exposed. In addition, whilst the majority of workers used some form of sun protection, only $9 \%$ were classified as adequately protected. This may suggest that sun exposure is not viewed as a serious hazard within workplaces, ${ }^{3}$ and points toward the need for formal workplace sun protection policies to be established. Past research has shown that formal policies lead to higher levels of compliance with sun protection guidelines, particularly when those policies are mandatory and subject to monitoring. ${ }^{29}$ Further education and training may also be required, particularly in those industries such as farming and construction where a number of workers are self-employed and so less likely to be subject to a formal sun protection policy.

Overall, this study represents an important addition to the scientific literature. A particular strength of this study is the use of a population-based approach, rather than focusing on specific occupations and industries, which allows for an examination of the characteristics of exposed workers and the circumstances of their exposure. This exposure information will be crucial to the future planning and prioritisation of strategies for skin cancer risk reduction in occupational settings. 


\section{References}

1. International Agency for Research on Cancer (IARC) [Internet]. A review of human carcinogens: Radiation: Volume 100D. Monographs on the Evaluation of Carcinogenic Risks to Humans 2012 [cited 2013 May 6]; Available from:

http://monographs.iarc.fr/ENG/Monographs/vol100D/index.php

2. Lucas R, McMichael T, Smith W, Armstrong B. Solar ultraviolet radiation: Global burden of disease from solar ultraviolet radiation. Geneva: World Health Organization; 2006.

3. Gies P, Wright J. Measured solar ultraviolet radiation exposures of outdoor workers in Queensland in the building and construction industry. Photochem Photobiol. 2003;78(4):3428.

4. Gies P, Roy C, Javorniczky J, Henderson S, Lemus-Deschamps L, Driscoll C. Global solar UV index: Australian measurements, forecasts and comparison with the UK. Photochem Photobiol. 2004;79(1):32-9.

5. Parisi AV, Wong JCF. Quantitative evaluation of the personal erythemal ultraviolet exposure in a car. Photodermatol Photo. 1998;14(1):12-6.

6. Cancer Council Victoria. Skin cancer and outdoor work: A guide for employers. Carlton: Cancer Council Victoria; 2007.

7. Kimlin MG, Parisi AV, Wong JCF. Quantification of personal solar UV exposure of outdoor workers, indoor workers and adolescents at two locations in Southeast Queensland. Photodermatol Photo. 1998;14(1):7-11.

8. Holman CDJ, Gibson IM, Stephenson M, Armstrong BK. Ultraviolet irradiation of human body sites in relation to occupation and outdoor activity: Field studies using personal UVR dosimeters. Clin Exp Dermatol. 1983;8(3):269-77. 
9. Parisi AV, Meldrum LR, Kimlin MG, Wong JCF, Aitken J, Mainstone JS. Evaluation of differences in ultraviolet exposure during weekend and weekday activities. Phys Med Biol. 2000;45(8):2253-62.

10. Hearne K, Makin J, Spittal M, Safe Work Australia. National Hazard Exposure Worker Surveillance: Exposure to direct sunlight and the provision of sun exposure controls in Australian workplaces. Canberra: Commonwealth of Australia; 2010.

11. Carey RN, Driscoll TR, Peters S, Glass DC, Reid A, Benke G, et al. Prevalence of Exposure to Occupational Carcinogens. Occup Environ Med in press.

12. Australian Bureau of Statistics. Socio-economic Indexes for Areas 2006. Canberra: Australian Bureau of Statistics; 2008.

13. Australian Bureau of Statistics. Australian Standard Geographical Classification. Canberra: Australian Bureau of Statistics; 2011.

14. Fernandez RC, Driscoll TR, Glass DC, Vallance D, Reid A, Benke G, et al. A priority list of occupational carcinogenic agents for preventative action in Australia. Aust N Z J Public Health 2012;36(2):111-5.

15. Australian Radiation Protection and Nuclear Safety Agency. Occupational exposure to ultraviolet radiation. Canberra: Commonwealth of Australia; 2006.

16. Fritschi L, Friesen MC, Glass D, Benke G, Girschik J, Sadkowsky T. OccIDEAS: Retrospective occupational exposure assessment in community-based studies made easier. Journal of Environmental Public Health. 2009;2009:957023.

17. Australian Bureau of Statistics. Australian and New Zealand Standard Classification of Occupations, first edition. Canberra: Australian Bureau of Statistics; 2006.

18. StataCorp LP. Stata version 12. College Station, TX.

19. Australian Bureau of Statistics. Census of Population and Housing. Canberra: Australian Bureau of Statistics; 2011. 
20. Australian Bureau of Meteorology [Internet]. Average solar ultraviolet (UV) index. 2012 [cited 201315 May]; Available from: www.bom.gov.au/jsp/ncc/climate_averages/uvindex/index.jsp

21. Peters CE, Nicol AM, Demers PA. Prevalence of exposure to solar ultraviolet radiation (UVR) on the job in Canada. Canadian Journal of Public Health. 2012;103(3):2236.

22. Young C, Rushton L, British Occupational Cancer Burden Study Group.

Occupational cancer in Britain: Skin cancer. Brit J Cancer. 2012;107:S71-5.

23. Blumthaler M, Ambach W. Solar UVB-albedo of various surfaces. Photochem Photobiol. 1988;48(1):85-8.

24. Lester RA, Parisi AV. Spectral ultraviolet albedo of roofing surfaces and human facial exposure. Int J Environ Health Res. 2002;12(1):75-81.

25. Diffey BL. Sources and measurement of ultraviolet radiation. Methods. 2002;28(1):413.

26. Glanz K, Buller DB, Saraiya M. Reducing ultraviolet radiation exposure among outdoor workers: State of the evidence and recommendations. Environ Health-Glob. $2007 ; 6: 22-32$.

27. Rosenthal FS, Phoon C, Bakalian AE, Taylor HR. The ocular dose of ultraviolet radiation to outdoor workers. Invest Ophth Vis Sci. 1988;29(4):649-56.

28. Shoveller JA, Lovato CY, Peters L, Rivers JK. Canadian national survey on sun exposure and protective behaviours: Outdoor workers. Canadian Journal of Public Health. 2000;91(1):34-5.

29. Australian Safety and Compensation Council. Guidance note for the protection of workers from the ultraviolet radiation in sunlight. Canberra: Commonwealth of Australia 2008. 


\section{Figure legend}

Fig. 1 Annual average Australian UV index 


\section{Tables}

Table 1. Adjusted odds ratios (OR) and 95\% confidence intervals (CI) for association between demographic characteristics and exposure to solar ultraviolet radiation

Demographic Characteristic Exposed Unexposed Adjusted $^{\mathrm{a}}$

$$
\mathrm{n}(\%)
$$

$\mathrm{n}(\%)$

OR $(95 \% \mathrm{CI})$

\section{Gender}

Male

963 (87.6)

$1803(46.3)$

1.0

Female

137 (12.4)

$2090(53.7)$

$0.3(0.2-0.4)$

\section{Age Group}

18-34

194 (17.7)

$553(14.3)$

1.0

$35-54$

$612(55.8)$

$2376(61.7)$

$0.8(0.6-1.1)$

55-65

$291(26.5)$

$925(24.0)$

$0.9(0.6-1.2)$

\section{Country of birth}

Australia

925 (84.2)

3043 (78.3)

1.0

Other

$174(15.8)$

845 (21.7)

$0.9(0.7-1.2)$

\section{Language at home}

English

$1090(99.1)$

$3806(97.8)$

1.0

Other

$10(0.9)$

$86(2.2)$

$1.3(0.5-3.2)$

\section{Highest education level}

High school or less

$522(47.5)$

$1321(34.1)$

1.0

Trade certificate or diploma

$419(38.1)$

$973(25.1)$

$1.0(0.8-1.3)$

Bachelor degree or higher

$158(14.4)$

$1585(40.8)$

$0.6(0.5-0.9)$

Socioeconomic status ${ }^{b}$

$p=0.167$

Fifth quintile (Highest)

$198(18.0)$

$1168(30.0)$

1.0

Fourth

$252(22.9)$

$996(25.6)$

$1.2(0.9-1.6)$ 


\begin{tabular}{lccc}
\hline Third & $258(23.5)$ & $761(19.5)$ & $1.3(0.9-1.8)$ \\
Second & $253(23.0)$ & $610(15.7)$ & $1.3(0.9-1.9)$ \\
First quintile (Lowest) & $139(12.6)$ & $358(9.2)$ & $1.6(1.1-2.5)$ \\
Remoteness & & & $p<.001$ \\
Major city & $487(44.3)$ & $2541(65.3)$ & 1.0 \\
Inner regional & $376(34.2)$ & $983(25.2)$ & $1.5(1.1-1.9)$ \\
Outer regional & $198(18.0)$ & $319(8.2)$ & $1.9(1.4-2.8)$ \\
Remote/very remote & $39(3.5)$ & $50(1.3)$ & $2.7(1.3-5.6)$ \\
\hline
\end{tabular}

${ }^{\text {a }}$ Adjusted for occupational group and all other variables in the model

${ }^{\mathrm{b}}$ From Socio-Economic Index for Areas Index of Relative Socio-economic Disadvantage (SEIFA IRSD)

${ }^{\mathrm{c}}$ From Australian Standard Geographical Classification Accessibility/Remoteness Index of Australia (ARIA+) 
Table 2. Number and percentage of total sample of respondents $(\mathrm{N}=4,993)$ exposed to solar ultraviolet radiation by occupational group and gender ${ }^{\text {a }}$

\begin{tabular}{|c|c|c|c|c|c|c|}
\hline \multirow[t]{2}{*}{ Occupational Group $^{b}$} & \multicolumn{2}{|r|}{ Overall } & \multicolumn{2}{|r|}{ Males } & \multicolumn{2}{|r|}{ Females } \\
\hline & $\mathrm{n}$ & $\%{ }^{\mathrm{c}}, 95 \% \mathrm{CI}^{\mathrm{d}}$ & $\mathrm{n}$ & $\%^{\mathrm{c}}, 95 \% \mathrm{CI}^{\mathrm{d}}$ & $\mathrm{n}$ & $\%^{\mathrm{c}}, 95 \% \mathrm{CI}^{\mathrm{d}}$ \\
\hline Farmers & 117 & $97.5,92.9-99.1$ & 90 & $97.8,92.4-99.4$ & 27 & $96.4,82.3-99.4$ \\
\hline Painters & 22 & $95.6,79.0-99.2$ & 22 & $95.6,79.0-99.2$ & - & - \\
\hline Plumbers & 65 & $94.2,86.0-97.7$ & 65 & $94.2,86.0-97.7$ & - & - \\
\hline Heavy Vehicle Drivers & 133 & $89.3,83.3-93.3$ & 128 & $90.1,84.1-94.0$ & 5 & $71.4,35.9-91.8$ \\
\hline Animal/Horticultural & 82 & $89.1,81.1-94.0$ & 66 & $97.1,89.9-99.2$ & 16 & $66.7,46.7-82.0$ \\
\hline Handypersons & 33 & $86.8,72.7-94.2$ & 29 & $87.9,72.3-95.2$ & 4 & $80.0,37.5-96.4$ \\
\hline Electrical Workers & 89 & $76.1,67.6-82.9$ & 86 & $76.1,67.5-83.0$ & 3 & $75.0,30.0-95.4$ \\
\hline Automobile Drivers & 47 & $74.6,62.7-83.7$ & 32 & $74.4,59.8-85.1$ & 15 & $75.0,53.1-88.8$ \\
\hline Construction Workers & 40 & $72.7,59.8-82.7$ & 40 & $74.1,61.1-83.4$ & - & - \\
\hline Engineers & 66 & $72.5,62.6-80.6$ & 61 & $72.6,62.2-81.0$ & 5 & $71.4,35.9-91.8$ \\
\hline Outdoor Work NEC & 21 & $72.4,54.3-85.3$ & 20 & $76.9,57.9-89.0$ & 1 & $33.3,6.1-79.2$ \\
\hline Warehousing & 22 & $68.7,51.4-82.0$ & 22 & $75.9,57.9-87.8$ & - & - \\
\hline Miners & 15 & $65.2,44.9-81.2$ & 15 & $68.2,47.3-83.6$ & - & - \\
\hline Carpenters & 56 & $63.6,53.2-72.9$ & 56 & $65.9,55.3-75.1$ & - & - \\
\hline Vehicle Trades & 34 & $42.0,31.8-52.8$ & 34 & $43.6,33.1-54.6$ & - & - \\
\hline Emergency Workers & 25 & $41.0,29.5-53.5$ & 21 & $38.2,26.5-51.4$ & 4 & $66.7,30.0-90.3$ \\
\hline Passenger Transport & 14 & $35.9,22.7-51.6$ & 13 & $41.9,26.4-59.2$ & 1 & $12.5,2.2-47.1$ \\
\hline Machine Operators & 24 & $34.8,24.6-46.5$ & 21 & $37.5,26.0-50.6$ & 3 & $23.1,8.2-50.3$ \\
\hline Scientists & 25 & $31.6,22.4-42.5$ & 18 & $48.6,33.4-64.1$ & 7 & $16.7,8.3-30.6$ \\
\hline Metal Workers & 20 & $19.4,12.9-28.1$ & 20 & $19.8,13.2-28.6$ & - & - \\
\hline Hospitality & 11 & $18.3,10.6-29.9$ & 2 & $9.5,2.6-28.9$ & 9 & $23.1,12.6-38.3$ \\
\hline
\end{tabular}




\begin{tabular}{|c|c|c|c|c|c|c|}
\hline $\begin{array}{l}\text { Other Health } \\
\text { Professionals }\end{array}$ & 10 & $9.4,5.2-16.5$ & 9 & $17.0,9.2-29.2$ & 1 & $1.9,0.3-9.9$ \\
\hline $\begin{array}{l}\text { Health/Personal } \\
\text { Support }\end{array}$ & 15 & $6.4,3.9-10.4$ & 3 & $6.7,2.3-17.9$ & 12 & $6.4,3.7-10.8$ \\
\hline Printers & 1 & $5.6,0.1-25.8$ & - & - & 1 & $25.0,4.6-69.9$ \\
\hline Office Workers & 106 & $4.5,3.7-5.4$ & 87 & $7.5,6.1-9.2$ & 19 & $1.6,1.0-2.5$ \\
\hline Food Factory & 1 & $2.6,0.5-13.5$ & 1 & $3.1,0.6-15.7$ & - & - \\
\hline Cleaners & 2 & $2.5,0.7-8.6$ & 2 & $6.4,1.8-20.7$ & - & - \\
\hline Teachers & 4 & $0.9,0.4-2.4$ & - & - & 4 & $1.3,0.5-3.3$ \\
\hline
\end{tabular}

${ }^{a}$ Occupational groups presented in descending order of exposure prevalence

${ }^{\mathrm{b}}$ No solar ultraviolet radiation exposure was observed for nurses or food service workers, and so these groups have been excluded

${ }^{\mathrm{c}}$ Percentage represents the percentage of workers from that occupational group assessed as being exposed to solar ultraviolet radiation

d $95 \%$ confidence interval of the proportion 